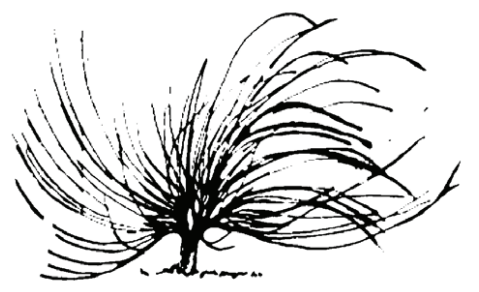

\title{
Simón Rodríguez y José Martí: rasgos iniciales de pedagogía crítica Latinoamericana
}

\author{
Diana López ${ }^{1}$ \\ Universidad Pedagógica Nacional de Colombia \\ Bogotá, Colombia \\ dianapraxis@gmail.com
}

\begin{abstract}
Resumen
La reflexión que se presenta a continuación expone algunas consideraciones sobre las principales ideas pedagógicas construidas en el siglo XIX por pensadores latinoamericanos y su relación con el espíritu de posteriores corrientes pedagógicas americanas, que desembocan en la construcción de las pedagogías críticas, comprendiendo las distancias contextuales que les han dado origen, mostrando la vinculación de un pensamiento regional-original que comienza con la independencia y se va enriqueciendo, profundizando en la posteridad.

Algunas de las preguntas que originan este escrito, que guían los variados y valiosos esfuerzos investigativos en el campo de la educación y la pedagogía son: ¿Por qué se puede considerar el pensamiento de José Martí y de Simón Rodríguez como el inicio de lo que hoy se concibe como pedagogías críticas? ¿Por qué es posible afirmar que allí, en el pensamiento de estos latinoamericanos subyacen los inicios de las pedagogías críticas? Se hace un análisis acerca de cuáles son los fundamentos o pilares
\end{abstract}

Recibido: 28 de julio de 2011 - Aprobado: 26 de marzo de 2012

1 Filósofa de la Universidad Nacional de Colombia, Magister en Educación de la Universidad Pedagógica Nacional de Colombia. Socia de la corporación Escuela Pedagógica Experimental, asesora pedagógica en Colegios Públicos. Forma parte del grupo de investigación sobre Pedagogías Emancipadoras Nuestroamericanas del Centro Cultural de la Cooperación de Bogotá. 
de las pedagogías críticas y qué coincidencias existen con la teoría expuesta por Simón Rodríguez y José Martí.

Palabras clave: Pensamiento y pedagogía Latinoamericana, Pedagogías Críticas.

\begin{abstract}
The reflection is shown below, presents some considerations about the main educational ideas $n$ the nineteenth century built by American thinkers and their relationship with the spirit of later American educational streams that flow into the construction of critical pedagogy, understanding the contextual distance have given origin, showing the linkage of a regional-original thought that begins with independence and is enriching, deepening posterity. Some of the questions that cause this writing, the varied and valuable guide research efforts in the field of education and pedagogy are: Why can consider the thought of José Martí and Simón Rodríguez as the beginning of what today is conceived as critical Pedagogies?, why it can be said that there, in thinking of these early American underlie critical pedagogies? An analysis of what are the foundations of critical pedagogies and those similarities exist with the theory advanced by Simón Rodríguez and José Martí.
\end{abstract}

Keywords: Latin American Thought and pedagogy, critical pedagogy.

\title{
Introducción
}

"Es una verdad extraordinaria: El gran espíritu universal tiene una faz particular en cada continente. Así nosotros, con todo el raquitismo de un infante mal herido en la cuna, tenemos toda la fogosidad generosa, inquietud valiente y bravo vuelo de una raza original, fiera y artística."

José Martí: “Los Códigos Nuevos” 

co, no sólo se circunscribe al pensamiento influenciado por el método marxista, aunque para algunos será considerado de esta manera.

Esto significa que, si bien en el pensamiento de Simón Rodríguez y José Martí no se encuentran rasgos de la actual Pedagogía Crítica, se encuentran, en su teoría pedagógica, algunos fundamentos derivados del pensamiento liberal ilustrado, cercanos y próximos de la teoría crítica expresada por Kant. La idea bastante fuerte en sus escritos del pensamiento propio, del cultivo de la razón y de la autonomía de los estudiantes, "hombres libres" los llama Martí en Función de la Enseñanza (Martí,1963, p. 238); “es hombre o mujer de Razón, es persona mui Racional" diría Rodríguez en Inventamos o Erramos (Rodríguez, 2001, p. 16), los ubica fuertemente en esta corriente de libre pensadores que abogan por un periodo y una nación ilustrada.

En tal sentido, el asunto de la vinculación de Simón Rodríguez y José Martí a la corriente crítica de la pedagogía implica reflexionar, además de los rasgos liberales que se encuentran en sus obras, acerca de otros fundamentos y consideraciones propias de las pedagogías críticas, para lo cual se hace indispensable revisar las analogías o confluencias entre dichos fundamentos y el pensamiento pedagógico de los autores latinoamericanos ya referidos.

\section{¿Qué caracteriza una Pedagogía Crítica?}

Es difícil realizar un inventario que caracterice una Pedagogía Crítica, en principio, y como un elemento central de ella, no existe una única Pedagogía Crítica sino que existen pedagogías críticas que comparten algunos fundamentos, entre ellos, afirma Freire: 1) Debe haber una ubicación en la historia, es decir, que la educación está situada en un tiempo y un espacio específico que la define y la orienta. 2) Al momento de hacer una reflexión y una propuesta pedagógica, se hace necesario realizar una lectura juiciosa del contexto en el que se encuentra ubicado un proceso educativo, pero no sólo en la perspectiva de realizar un diagnóstico de la realidad, sino para incorporar ese contexto como parte del proceso de transformación de la realidad, en 
ese sentido. 3) Se contempla -se teoriza- para la acción -la praxis- que no es otra cosa que la transformación de las condiciones materiales de quienes están involucrados en el proceso educativo ya que la educación es un hecho social, lo que hace que la transformación de la educación propenda por una transformación de realidades concretas que se dan en ese ámbito social, económico y político; en este sentido, la pedagogía es un ejercicio político para la transformación de la realidad. De tal manera, podemos observar como elemento sustantivo de una Pedagogía Crítica, una lectura del contexto, crítica en la perspectiva de la acción para la transformación de la realidad donde se ubica.

Otro elemento sustancial que se haya en el fondo de la reflexión de las pedagogías críticas es la imperiosa necesidad de contribuir a eliminar las causas de la opresión en los seres humanos. Freire planteará que el problema central de toda pedagogía transformadora es la dominación de unos sobre otros, en cualquier condición, no solamente económica sino también cultural, de esa manera, la pedagogía se convierte en un proceso dialógico y liberador de los oprimidos: "De ahí la necesidad que se impone de superar la situación opresora. Esto implica el reconocimiento crítico de la razón de esta situación, a fin de lograr a través de una acción transformadora que incida sobre la realidad, la instauración de una situación diferente, que posibilite la búsqueda del ser más"(Freire, 1980, p. 28).

De otra parte, encontramos en La Pedagogía Crítica y la Pragmática de la Justicia de McLaren, unos principios que él denomina comunes en la Pedagogía Crítica:

1. Las Pedagogías deben constituir una forma de crítica social y cultural.

2. Todo conocimiento está fundamentalmente mediado por relaciones lingüísticas social e históricamente constituidas.

3. Los individuos mantienen con el resto de la sociedad una relación de sinécdoque, por medio de tradiciones de mediación (la familia, los amigos, la religión, la educación formal, la cultura popular, etc.).

4. Los hechos sociales nunca pueden ser aislados del dominio de los valores o de las formas de producción e inscripción ideológicas.

5. Las relaciones entre concepto y objeto y significante y significado no son inherentemente estables ni están trascendentalmente 
fijadas, viéndose a menudo, mediadas por los circuitos de producción capitalista, el consumo y las relaciones sociales.

6. El lenguaje desempeña un papel central en la formación de la subjetividad (tanto consciente como inconsciente).

7. En toda sociedad, ciertos grupos son innecesaria y a menudo injustamente privilegiados con respecto a otros, y si bien la razón de tal privilegio puede variar ampliamente, la opresión que caracteriza a las sociedades contemporáneas se ve fuertemente asegurada cuando los subordinados aceptan su estatus social como un elemento natural, necesario, inevitable o heredado de la fatalidad histórica.

8. La opresión tiene muchas facetas, y concentrarse sólo en una de ellas a expensas de las demás (por ejemplo, en la opresión de clase, ignorando el racismo) a menudo omite u oculta sus interrelaciones.

9. Tenemos por delante un mundo nunca visto de relaciones sociales, donde el poder y la opresión no puedan entenderse meramente en términos de un cálculo irrefutable de sentido ligado a condiciones de causa y efecto.

10. La dominación y la opresión están implicadas en la contingencia radical del desarrollo social y en nuestras respuestas a él.

11. Las prácticas de investigación dominantes están general e involuntariamente implicadas en la reproducción del sistema de clases, de razas y la opresión de género.(McLaren citado por Leonardo, 2007, p. 81).

Por su parte, Giroux en su libro Los profesores como Intelectuales define la pedagogía crítica como una intervención importante en la lucha por reestructurar las condiciones ideológicas y materiales de la sociedad en general, en pro de una sociedad democrática. Ubica a los profesores como intelectuales cuando combinan la reflexión y la acción con el fin de potenciar a los estudiantes con las habilidades y conocimientos para luchar contra las injusticias y convertirse en actores críticos; igualmente afirma que pueden desempeñarse como parte de una red pedagógica de solidaridad para mantener vivo el hecho histórico y popular. También considera que la Pedagogía Crítica se propone ver la escuela como un escenario de la cultura: 
Las Escuelas son lugares sociales constituidos por un conjunto de culturas dominantes y subordinadas.(...) Los maestros deben tomar conciencia de la cultura dominante y cómo funciona, deberían luchar por sus propias experiencias, cultura, etc. (..) Se deben desenmascarar las concepciones de poder que subyacen en los currículos, tomar en cuenta a los estudiantes y su saber. (...) Nuestra preocupación primaria es el problema educativo de lo que significa enseñar a los estudiantes a pensar críticamente, a afirmar sus propias experiencias y a comprender la necesidad de luchar individual y colectivamente por una sociedad más justa(Giroux, 2000, p.75).

En tal sentido, plantea la formación como un proceso de concienciación de los estudiantes en pro de la construcción de un pensamiento propio, ligado tanto al reconocimiento de sus condiciones materiales y sus experiencias como productoras de conocimiento, como a las posibilidades de transformación de aquellas condiciones de dominación que le impiden ser y reconocer-se; dado que el currículo actual manifiesta las relaciones entre sociedad y escuela, de tal manera, que los profesores sólo transmiten actitudes, normas y creencias incuestionadas, además confirman determinadas formas de desarrollo cognitivo y disposicionalque afianza las formas de opresión institucional que están ligadas directamente con el sistema capitalista que los oprime.

Otros elementos que se manifiestan tanto en el pensamiento de Giroux como en el de McLaren y que podrían identificar a las Pedagogías Críticas son la lucha contra el sexismo, el racismo y cualquier forma de discriminación, así como, la lucha contra el desequilibrio ambiental; todo esto basado en múltiples teorías contemporáneas que reconocen en estas manifestaciones más situadas, el inicio de una disputa contra la opresión y las desigualdades sociales. En tal sentido, la consideración central de toda pedagogía crítica se circunscribe en que es necesario que lo pedagógico sea más político, a su vez que lo político sea cada vez más pedagógico.

Las propuestas de Rodríguez y Martí, que están ligadas directamente a un proyecto de región, de nación y de sistema político para un continente que resurge luego de la conquista y la colonia, son quizá el primer elemento de conexión con el interés de las pedagogías críticas en relacionar y hacer interactuar lo político con lo pedagógico y viceversa. La firme convicción de que hay que comenzar algo nuevo a partir de 
lo que nos ha dejado el pasado es el inicio de una propuesta, que no se limita a ser política o pedagógica, se combina y se identifica en la dos, lo que permite pensar que allí hay un rasgo de pedagogía crítica.

\section{Simón Rodríguez y José Martí: ¿Pedagogos críticos?}

Rodríguez decía: "YO NO AMENAZO: Sólo pido, a mis contemporáneos, Una declaración, que me recomiende a la posteridad, como al primero que propuso, en su tiempo, medios seguros de reformar las costumbres, para evitar revoluciones-empezando Por la ECONOMIA social, con una EDUCACIÓN POPULAR'(Rodríguez, 1988, p. 121), a primera vista se puede considerar que Rodríguez pensaba todo lo contrario a lo planteado por las Pedagogías Críticas, ya que se puede entender como un deseo de domesticar a los pobladores de América para que no se revelaran, sin embargo, leyendo con más atención y en el contexto general del documento, lo que está planteando Rodríguez es que debe haber una educación para todo el mundo, una educación popular como él mismo la nombrara, y no una educación para las minorías, más adelante incluso, pedirá una educación de igual nivel para todos, una educación que reconozca a todos los americanos como ciudadanos con el derecho a educarse, en ese sentido, una educación para la resistencia y de rebelión frente a la dominación extranjera, porque si no se educa al ciudadano de la república en los nuevos valores, es posible que el pueblo se revele, lo que quiere decir, que tarde o temprano el pueblo reconozca que también es su derecho recibir educación y condiciones de igualdad.

En este escrito, Rodríguez inicia con una reflexión sobre lo popular, sobre la economía y la política para luego hacer una larga referencia al contexto de la época y proponer una liberación de la dependencia que se tiene de Europa, exhortando a la realización de un pensamiento propio: "Dejemos la Francia i veamos la AMÉRICA"invita con fuerza y luego dice que las sociedades tienden a vivir de un modo diferente al que han vivido: "Quieren vivir sin reyes y sin Congresos, no quieren tener amos ni TUTORES, quieren ser dueños de sus personas, de sus bienes y de su voluntad; sin que por eso se entiendan vivir como ANIMALES FEROCES, (que es lo que suponen los defensores del absolutismo manifiesto o paliado). QUIEREN gobernarse por la RAZÓN que es la autoridad de la naturaleza. RAZÓ, es figura abstracta de la facultad de pensar"(Rodríguez, 1988, pp.126-127), indicando que es necesario 
enseñar a pensar, a elaborar un pensamiento propio que logre construir la República y que logre desprendernos de la dependencia de Europa.

Sin duda, Rodríguez realiza un análisis del contexto de la época en cada escrito elaborado, manifestando de manera muy clara en el Extracto Sucinto de su Obra sobre la Educación Republicana una propuesta de educación para la construcción de una República. Jamás deja de lado las apreciaciones acerca de las desigualdades sociales que no dejan fomentar una cultura propia de una República y reconoce todo el tiempo las diferencias entre colonos e indios y su derecho a estudiar su lengua y reconocerla como propia, sin negar lo suyo, lo que manifiesta Rodríguez una abierta defensa de los derechos de los indígenas: "Castellano y Quichua. El primero es de obligación, y el segundo de conveniencia. El latín no se usa sino en la iglesia.-Apréndalo el que quiera ordenarse"(Rodríguez, 1988, p. 213).

Rodríguez además hace una vehemente crítica al dominio de otros países sobre América, de tal suerte que su posición es claramente independentista y rechaza toda forma colonial de sociedad, "La América debe ser original, si quiere copiar algo que sea la originalidad" dirá en Sobre Luces y Virtudes Sociales. Su búsqueda por una educación popular, para todos, muestra que en el fondo de su reflexión y de manera expresa, asuma una posición política contraria a la que se mantenía establecida a partir de la colonia, además critica fuertemente que se quiera iniciar la consolidación de las Repúblicas manteniendo ideas colonizadoras, en especial, manteniendo la idea de que la educación es para los que tienen dinero y pueden pagar educación privada, haciendo una defensa de la educación para todos y con una marcada intención de formar ciudadanos y no "reproductores" de letras muertas, aprendidas de memoria y sin ningún sentido ni provecho para la vida en la República:

OBEDECER CIEGAMENTE, es el principio que gobierna. Por eso hay tantos Esclavos - i por eso es Amo el primero que quiere serlo. Enseñen a los Niños a ser PREGUNTONES! Para que pidiendo el Por Qué, de lo que se les mande hacer, se acostumbren a obedecer...a la RAZÓN! No a la AUTORIDAD, como los LIMITADOS ni a la COSTUMBRE, como a los ESTÚPIDOS(Rodríguez, 2001, pp.26-27). 
Se evidencian entonces, elementos importantes de pensamiento y pedagogía crítica en Rodríguez, no sólo porque proponga una nueva forma de gobierno, insta a la industrialización y exija una separación de la dependencia extranjera, sino porque además propone a la educación, y a la enseñanza en particular, como un ejercicio de transformación social, ese es quizá el elemento más fuerte y que se puede observar más claramente en la carta escrita al Rector del colegio Latacunga: "Dar gritos y hacer ringorrangos no es aprender a leer ni a escribir. Mandar recitar, de memoria, lo que no se entiende, es hacer papagallos, para que...por la vida!...sean charlatanes"(Rodríguez, 1988, p. 207).

Otro elemento de coincidencia es su preocupación por la enseñanza y cómo preparar a los maestros para que sean profesionales, a los que se les reconozca como tales y quienes puedan asumir la formación adecuada de todos los niños en los primeros años. Además, los maestros deben asumir un rol protagónico en la sociedad y reconozcan el contexto, las necesidades y los saberes de los niños. "La enseñanza debe ser JENERAL i CONSTANTE, su IMPORTANCIA! Exige que haya, en ella, Maestros Sabios, Hábiles, Irreprensibles i...i...con VOCACIÓN! Para Enseñar'(Rodríguez, 2001, p. 52). Un maestro formador y no reproductor de letras muertas, sería una de las condiciones de Rodríguez para los nuevos maestros.

La importancia de la palabra, el lenguaje, el estudio de las lenguas propias y al final el significado de las palabras escritas, son otro elemento coincidente con los postulados de las pedagogías críticasya que su preocupación sobrepasa la idea de la Ilustración de educar a las nuevas generaciones a partir del estudio de las lenguas clásicas y de la lectura de clásicos europeos, para proponer el estudio del Quechua, el estudio del castellano a partir de su incorporación en la cultura, la escritura libre, no ligada de manera inminente a lecturas de clásicos sino dando rienda suelta a su imaginación, lo que ubica a Rodríguez por fuera de esa idea clásica del uso de la razón permanente o incluso para la obediencia. Por el contrario, buscando siempre la libertad a partir del quehacer en la escuela y en particular, a partir del lenguaje.

En todas estas consideraciones sobre la educación de los americanos, están ligadas de manera evidente en las elaboraciones de Rodríguez, la necesidad de formar ciudadanos de la República que se está conformando. En ello, Rodríguez hace énfasis todo el tiempo y subraya además que no puede pensarse en ese momento en una educación para otros fines que no sean el cultivo de la sociedad y la ciudadanía, lo 
que está en plena consonancia con el planteamiento de las Pedagogías Críticas, en cuanto a la importancia de ver la educación y la pedagogía como hechos políticos en escenarios concretos, en momentos históricos donde se requiere liberarse, no sólo desde el punto de vista económico sino también cultural y para ello se requiere de una educación liberadora que contribuya en la transformación de la sociedad.

"El hombre no es Ignorante, porque es POBRE, sino al contrario.

JENERALÍCESE la INSTRUCCIÓN de la INFANCIA

I habrá

LUCES i VIRTUDES SOCIALES

Luces i Virtudes hay...

Pero...

Lo que no es JENERAL, no es PÚBLICO - i lo que no es PÚBLICO, No es SOCIAL.

Si los Gobiernos llegaran a persuadirse, de que el primer deber, que les impone su Misión, es el de cuidar que no haya, en sus ESTADOS, Un SOLO INIDVIDUO!

Que Ignore sus DERECHOS i DEBERES SOCIALES, Habrían dado un GRAN PASO!

En la Carrera de la Civilización, que abre el siglo PRESENTE." (Rodríguez: 2001; p 30).

Un último elemento a considerar aquí y que resulta articulador entre el pensamiento emancipador y las pedagogías críticas es la importancia del trabajo en la educación. Su inquietud por una educación que enseñe a trabajar, a cultivar la tierra, a transformar la naturaleza y que a su vez tenga el reconocimiento formador y económico para quienes asisten a la escuela. Aunque no de manera directa, las Pedagogías Críticas han de reconocer que la escuela debe recrearse a partir de los contextos propios de los niños, a la construcción sociocultural que le es propia y seguramente allí, el trabajo, las actividades productivas, autogestionarias, que no dependan sino de la comunidad se han de considerar liberadoras y emancipadoras de la sociedad.

Por su parte Martí, contemporáneo de Rodríguez y su conciudadano latinoamericano, asume también la educación como un derecho de todos. "Una nación libre es el resultado de sus pobladores libres" (Martí, 1990, p. 39), reconoce la necesidad de la enseñanza obligatoria y de 
cómo los maestros deben ser los que vayan hacia sus estudiantes y no lo contrario; reconoce además la educación popular como una necesidad de dar educación a todo el pueblo, de la misma manera, reconoce la ineludible tarea de liberarse de la imposición de Norte América, es decir, es un hombre que lucha contra la imposición de unos sobre otros. Se destaca su preocupación por una educación contextual, que reconozca las necesidades y características de un pueblo original como el americano, sin estar buscando reproducir modelos foráneos o modas externas que no permiten una educación acorde con la construcción de una ciudadanía propia.

Si se preparase a los sudamericanos, no para vivir en Francia, cuando no son franceses, ni en los Estados Unidos, que es la más fecunda de estas modas malas, cuando no son norteamericanos, ni en los tiempos coloniales, cuando están viviendo ya fuera de la colonia, en competencia con pueblos activos, creadores, vivos, libres, sino para vivir en la América del Sur! . . Mata a su hijo en la América del Sur el que le da mera educación universitaria (Martí, 1963, p.24).

La educación ha de entenderse como elemento liberador, generador de principios y actuaciones autónomas que den prioridad a las condiciones particulares de América del Sur y que permitan el desarrollo de Repúblicas independientes con ciudadanos que le den firmeza y sostenibilidad a un proyecto político en construcción. "Ser bueno es el único modo de ser dichoso. Ser culto es el único modo de ser libre"(Martí, 1963, p. 288). De este modo la formación ha de propiciar en los americanos una voluntad de ser libres y de luchar porque esa libertad dé como resultado una independencia tanto individual como colectiva, es decir, emancipadora de la sociedad. "El remedio está en cambiar bravamente la instrucción primaria de verbal en experimental, de retórica en científica. (...) Hombres vivos, hombres directos, hombres independientes, hombres amantes,-eso han de hacer las escuelas, que ahora no hacen eso"(Martí, 1990, p. 16). Haciendo énfasis en la urgencia de cambiar las escuelas, de transformar la enseñanza para consolidar procesos pedagógico-políticos que den resultados en la vida tanto personal como social de los americanos.

En Martí también se hace referencia a la necesidad de educar para el trabajo. Enseñar en el hacer, en labrar la tierra, en trabajar con las manos en la escuela, ubicado como una necesidad para mejorar la educación y la sociedad: 
Los hombres necesitan conocer la composición, fecundación, transformaciones y aplicaciones de los elementos materiales de cuyo laboreo les viene la saludable arrogancia del que trabaja directamente en la naturaleza, el vigor del cuerpo que resulta del contacto con las fuerzas de la tierra, y la fortuna honesta y segura que produce su cultivo. (...) Y en campos como en ciudades, urge sustituir al conocimiento indirecto y estéril de los libros, el conocimiento directo y fecundo de la naturaleza (Martí, 1963, p. 292).

Tanto Rodríguez como Martí hacen una crítica muy fuerte de la escuela en su momento ya que mantiene los ejercicios de dominación y no forma generaciones de hombres libres, lo que indica en ellos una necesidad de transformar las prácticas educativas, en pro de generar pensamientos propios, identidades propias y gobiernos propios. Una clara postura política que los presenta ante la historia como los grandes pensadores de la sociedad y la política desde la reflexión sobre las necesarias transformaciones de la Educación y la Pedagogía; pero tal vez lo más importante de su aporte y que los acerca mucho a las pedagogías Críticas es su vida personal, comprometida con luchar por las transformaciones necesarias para un cambio social. Sus vidas, sus acciones y su pensamiento, los devela como los próceres del pensamiento latinoamericano y por qué no, las primeras aproximaciones a lo que se ha de denominar en su futuro no tan lejano, pedagogías críticas.

\section{Referencias bibliográficas}

Freire, P. (1980). Pedagogía del Oprimido. México: Siglo XXI.

Freire, P. (2007). La Educación como Práctica de la Libertad. Montevideo: Siglo XXI. Giroux, H. (2000). Los Profesores como Intelectuales. México: Siglo XXI

Huertas-Charles, L., Marc, P. (2007).De la Pedagogía Crítica a la Pedagogía de la Revolución. México: siglo XXI.

Martí. J.(1963). “La América, Nueva York, noviembre de 1884”. Reproducido en Obras completas. Volumen VI y VIII. La Habana: Editorial Nacional de Cuba, 1963. [Versión digital preparada por Marina Herbst.]

Martí, J. (1990).Ideario Pedagógico. La Habana: Pueblo y Educación.

Rodríguez, S. (1988). Inventamos o Erramos. Caracas: Monte Ávila.

Rodríguez, S. (2001). Obras completas. Tomo Iy II. Caracas: Presidencia de la República. 